\title{
Sketch Based Face Recognition: Forensic vs. Composite Sketches
}

\author{
Scott Klum, Hu Han, Anil K. Jain \\ Department of Computer Science and Engineering \\ Michigan State University, East Lansing, MI, U.S.A. \\ $\{$ klumscot, hhan, jain\}@cse.msu.edu
}

\author{
Brendan Klare \\ Noblis, Falls Church, VA, U.S.A \\ brendan.klare@noblis.org
}

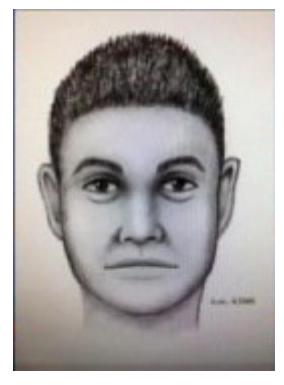

(a)

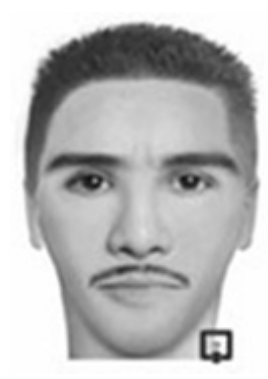

(c)

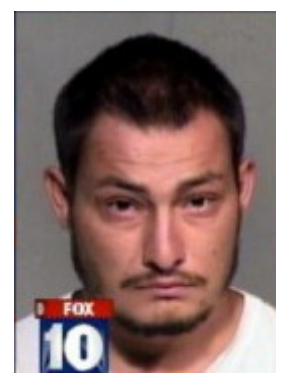

(b)

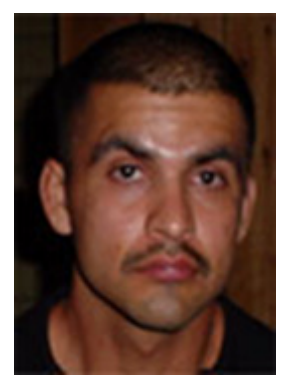

(d) made available to interested researchers; (ii) a comparison of holistic facial representations versus component based representations for sketch to mugshot matching; and (iii) an analysis of the effect of filtering a mugshot gallery using three sources of demographic information (age, gender and race/ethnicity). Our experimental results show that composite sketches are matched with higher accuracy than forensic sketches to the corresponding mugshots. Both of the face representations studied here yield higher sketch to photo matching accuracy compared to a commercial face matcher.

\section{Introduction}

Facial sketches are commonly used in law enforcement to assist in identifying suspects involved in a crime when no facial image of the suspect is available at the crime scene

\footnotetext{
${ }^{1}$ http: //www.askaforensicartist. com/phoenix-pol ice-sketch-leads-to-arrest-of-kidnapper/

${ }^{2}$ http://www.facesid.com/mediacenter_frontline_ stories.html
}

Figure 1: Examples of facial sketches $(\mathrm{a}, \mathrm{c})$ used in successfully apprehending the suspects whose mugshots were in the database $(b, d)$. (a, b): A man accused of attempting to kidnap a young girl was captured based on his forensic sketch ${ }^{1}$. (c, d): A composite sketch created using the software FACES [1] assisted in the capture of a Florida man who brutally attacked and attempted to abduct several young women ${ }^{2}$.

(e.g., from a surveillance camera or a mobile phone). After a sketch of a suspect's face is created, authorities disseminate the sketch with the hope that someone will recognize the individual and provide pertinent information leading to an arrest. Facial sketches are particularly valuable when eyewitnesses' or victim's descriptions are the only form of evidence available [2]. Unfortunately, this process is inefficient and does not leverage all available resources, in particular, the extensive mugshot databases maintained by law enforcement agencies. Successful techniques for automatically matching facial sketches to mugshots will improve the 

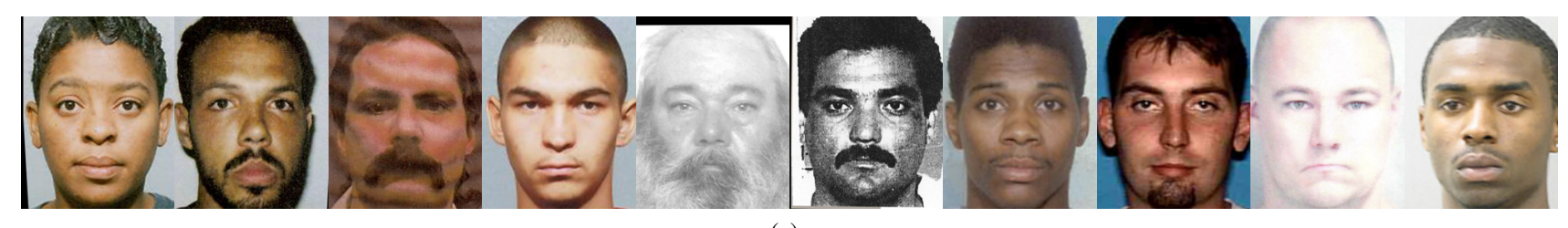

(a)
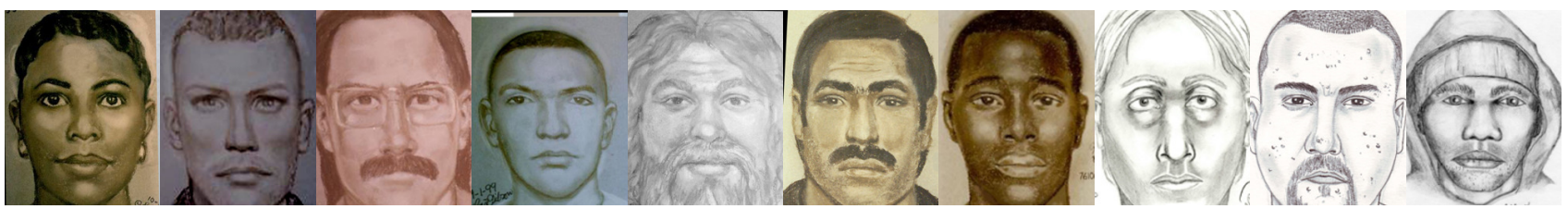

(b)
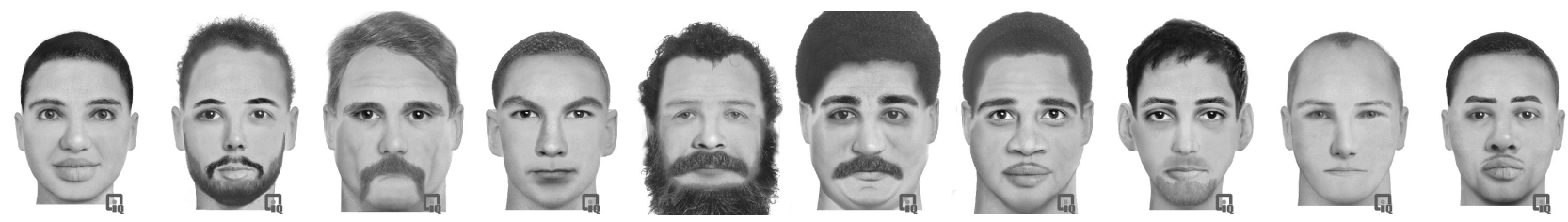

(c)

Figure 2: Examples of (a) mugshots and corresponding (b) forensic sketches and (c) composite sketches of ten different subjects used in our study. These mugshots and forensic sketches are available in the public domain. The composite sketches were drawn by the authors using the software FACES [1] based on a description provided by a volunteer (acting the role of a witness).

effectiveness of facial sketches and allow for a faster apprehension of such suspects. For the remainder of this paper, we use mugshot and photograph interchangeably.

Facial sketches used in law enforcement can be divided into two categories:

(i) Forensic sketches: Facial sketches drawn by forensic artists based on the description provided by a witness (Fig. 1 (b)). Forensic sketches have been used in criminal investigations dating as far back as the 19th century [3].

(ii) Composite sketches: Facial sketches created using software kits which allow an operator to select various facial components (Fig. 1 (c)). Composite sketches have become a popular and more affordable alternative to forensic sketches. In fact, $80 \%$ of law enforcement agencies reported using some form of software to create facial sketches of suspects [3].

Whereas forensic sketch artists typically require a few years of training to become proficient in drawing sketches, only a few hours of training are required before a police officer becomes proficient in using composite sketch software. Irrespective of the quality and capability of the software, most composite software kits rely on choosing a set of facial components (e.g., eyes, nose, mouth) based on the information contained in the suspect's description.

While several methods that match viewed ${ }^{3}$ and foren- sic sketches to mugshots have been reported in the literature $[4,5,6,7,8,9,10]$, far fewer methods exist for automatic matching of composite sketches to mugshots [7,11]. In all the previous studies reported on composite sketches, sketches were created while the operator was viewing the mugshot. This type of viewed sketch does not accurately reflect the creation of composite sketches for use in criminal investigations because the mugshot of the suspect is unknown or unavailable. Indeed, there would be no need to draw the sketch if we knew the suspect and had his mugshot.

Criminal psychology literature contains a few studies that compare the human accuracy in matching forensic sketches and composite sketches to mugshots. Frowd et al. [12] conclude that forensic sketches are more accurately recognized than composite sketches in a rank-one naming task with a two day delay (time difference between a subject viewing the suspect and providing suspect's description). When this time difference is relatively short (3-5 hours), $20 \%$ of the composite sketches drawn were correctly recognized $[13,14]$.

To our knowledge, no study has been reported comparing the accuracy of automatic face recognition systems in matching forensic sketches versus composite sketches. To facilitate this comparison, we have created a new database containing both modalities of facial sketches with corre-

\footnotetext{
${ }^{3}$ Many studies on facial sketch to photograph matching have relied on viewed sketches in which the facial sketch is drawn by hand while viewing the photograph. We ignore these types of sketches because they are not relevant to forensic and law enforcement scenarios.
} 


\begin{tabular}{|c|c|c|c|}
\hline & Publication & Approach & Limitations \\
\hline $\begin{array}{l}\vec{D} \\
\sum_{0}^{0} \\
;\end{array}$ & $\begin{array}{l}\text { Tang and Wang [4] } \\
\text { Liu et al. [5] } \\
\text { Gao et al. [15] } \\
\text { Wang and Tang [8] } \\
\text { Lin and Tang [6] } \\
\text { Zhang et al. [16] }\end{array}$ & $\begin{array}{l}\text { Photo-to-sketch conversion using eigen- } \\
\text { transform } \\
\text { Photo-to-sketch conversion using locally } \\
\text { linear embedding } \\
\text { Photo-to-sketch conversion using embed- } \\
\text { ded hidden Markov model } \\
\text { Photo-to-sketch conversion using multi- } \\
\text { scale Markov random field model } \\
\text { Common discriminant feature extraction } \\
\text { PCA based algorithm }\end{array}$ & $\begin{array}{l}\text { Viewed sketches are not useful in law } \\
\text { enforcement and forensics applications. } \\
\text { Conversion methods are often solving a } \\
\text { more difficult problem than the recognition } \\
\text { task. }\end{array}$ \\
\hline 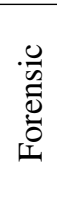 & $\begin{array}{l}\text { Uhl and Lobo [17] } \\
\text { Klare and Jain [9] } \\
\text { Bhatt } \text { et al. [18] }\end{array}$ & $\begin{array}{l}\text { Photometric standardization } \\
\text { SIFT and MBLP feature descriptors with } \\
\text { local-feature based discriminant analysis } \\
\text { Multi-scale circular Weber's local descrip- } \\
\text { tor }\end{array}$ & $\begin{array}{l}\text { Composite sketches, which are widely used } \\
\text { in law enforcement, were not considered. }\end{array}$ \\
\hline 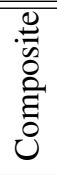 & $\begin{array}{l}\text { Yuen and Man [7] } \\
\text { Han et al. [11] }\end{array}$ & $\begin{array}{l}\text { Point distribution model and geometrical } \\
\text { relationship } \\
\text { Component based representation using } \\
\text { MLBP descriptors }\end{array}$ & $\begin{array}{l}\text { Composite sketches were created while } \\
\text { viewing the photograph. Forensic sketches } \\
\text { were not considered. }\end{array}$ \\
\hline & & & Contributions \\
\hline & Proposed & $\begin{array}{l}\text { Both forensic and composite sketches are } \\
\text { studied using two representations methods. }\end{array}$ & $\begin{array}{l}\text { A new database is created containing both } \\
\text { forensic and composite sketches for } 75 \\
\text { different mugshots. Demographic } \\
\text { information is used to improve the } \\
\text { matching performance. }\end{array}$ \\
\hline
\end{tabular}

Table 1: Previous work related to matching facial sketches to photographs. Published papers have been divided into three categories based on the type of sketch used (viewed, forensic and composite). As mentioned earlier, viewed sketches are not used in law enforcement.

sponding mugshots (available at http://biometrics .cse.msu.edu/pubs/databases.html). The primary goal of this paper is to compare the performance of two different automatic face recognition systems (holistic and component based) with respect to matching forensic sketches and composite sketches to mugshots. Demographic information of subjects (e.g., gender, age range, and race/ethnicity) is further utilized to filter the gallery and improve the matching performance. We also used the commercial face matching system FaceVACS [19] to establish a baseline recognition accuracy between facial sketch and photo.

\section{Related Work}

Automated face matching between two facial photographs is a widely studied problem in computer vision, pattern recognition, and biometrics [20]. However, matching facial sketches to photographs is a more challenging problem with only a limited amount of published work $[4,5,6,7,8,9,10,11]$. Of these, most studies have used viewed sketches drawn while viewing the mugshot photograph. Further, the studies that considered opera- tional forensic sketches did not address the use of composite sketches.

To our knowledge, only two previous studies focused on automatic face recognition systems using composite sketches. The first used a combination of local and global features to represent sketches [7], but it required user input in the form of relevance feedback in the matching or recognition phase. Further, the authors in [7] used a small gallery in their experiments (300 facial photographs). The method proposed by Han et al. [11], used a component based approach to match facial sketches to mugshots. While Han et al. used a large gallery and created a method that is fully automatic, the composite sketches used were created while viewing the mugshot photograph (viewed composite sketches) which does not reflect operational scenarios.

Our work uses forensic sketches from criminal investigations and composite sketches created using descriptions from volunteers given two days after viewing a mugshot, mimicking a witness of an actual crime scene. Furthermore, we compare the recognition accuracy of composite sketches versus forensic sketches using two different automated face recognition systems and examine the effect of filtering the 


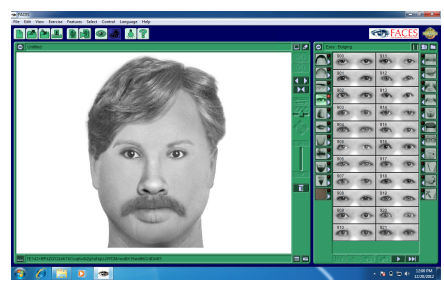

(a)

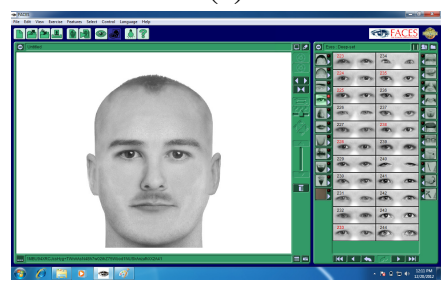

(c)

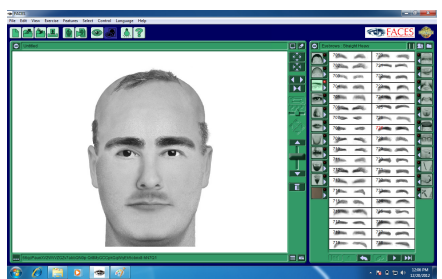

(b)

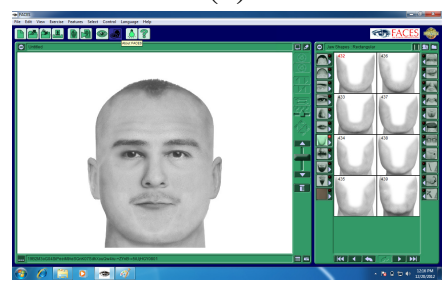

(d)

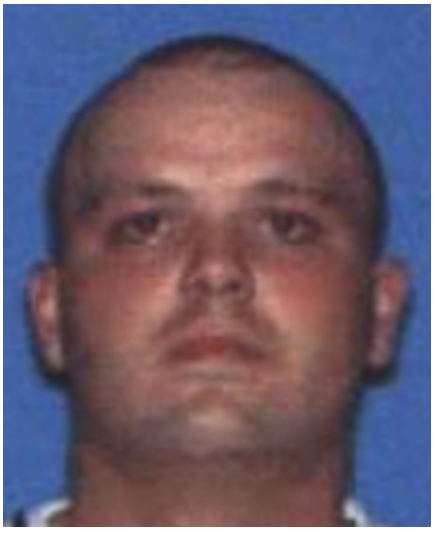

(e)

Figure 3: Creating a composite sketch with the commercial software FACES [1]. Initially, a face is generated randomly using an option provided in the software (a). The operator then modifies it based the description provided by the volunteer (witness) (b, c) until a satisfactory sketch (as indicated by the volunteer) is obtained (d). For this example, the mugshot of the suspect that the volunteer saw two days prior to providing the description is shown in (e).

gallery with demographic information of the suspect provided by the volunteer. A summary of related work can be found in Table 1.

\section{Constructing a facial sketch}

Despite the numerous public databases available containing facial images, to our knowledge, none exist that have both forensic sketches and composite sketches for the same mugshot. Therefore, we created a set of composite sketches for a subset of an existing database of forensic sketch and mugshot photo pairs which were used by Klare and Jain [10]. Fig. 2 shows examples of mugshots and forensic and composite facial sketches used in our study.

\subsection{Forensic sketches}

All forensic sketches used in our study were created by forensic sketch artists for real-world criminal investigations. To create a forensic sketch, an artist draws a face based on descriptions provided by either one or multiple eye witnesses. For this type of facial sketch, the time between observation and recall by a witness varies depending on the circumstances. Of the 75 total forensic sketches used in our experiments, 50 were drawn by Lois Gibson [21] and the remaining 25 were drawn by forensic artists employed by the Michigan State Police.

\subsection{Composite sketches}

A number of software systems exist to create composite sketches: E-FIT [22], EvoFit [23], FACES [1], IdentiKit [24], Mac-a-Mug [25], and Photo-Fit [25]. Of these, Identi-Kit and FACES are most widely used by law enforcement agencies in the United States [3]. Both IdentiKit and
FACES allow users to choose from a set of candidate components or features (i.e. eyes, mouth, nose). FACES provides a larger number of features and options, and it has been observed to be subjectively more accurate than IdentiKit [11]. For these two reasons, we used FACES to create composite sketches for our study.

To create the composite sketches, we used a procedure designed to mimic real-world composite sketch synthesis. Volunteers (adults ranging from 20-40 years of age) were asked to view a mugshot of a suspect for one minute. Two days later they were asked to describe the mugshot to the FACES software operator (one of the authors of this paper) who had not seen the mugshot. Volunteers also provided demographic information to the best of their ability (gender, race/ethnicity, age range). During the description process, the FACES operator used a cognitive interview technique [26] to enhance the volunteer's memory of the suspect's facial features in the mugshot. To reduce the problem of operator contamination, in which previously created composites influence the creation of the current composite, a random composite face was generated initially then modified based on the volunteer's description (Fig. 3). It is worth noting that certain limitations exist when creating composite sketches. For example, it is difficult to achieve certain types of shading and skin texture in the composite sketch. The high variability in different suspects' hairstyles versus those contained in the menu option of FACES makes picking the correct hairstyle difficult. In total, 75 composite sketches were synthesized, each taking 30 minutes to create on average. This database of composite sketches, forensic sketches, and and mugshots is available at http://biom etrics.cse.msu.edu/pubs/databases.html. 


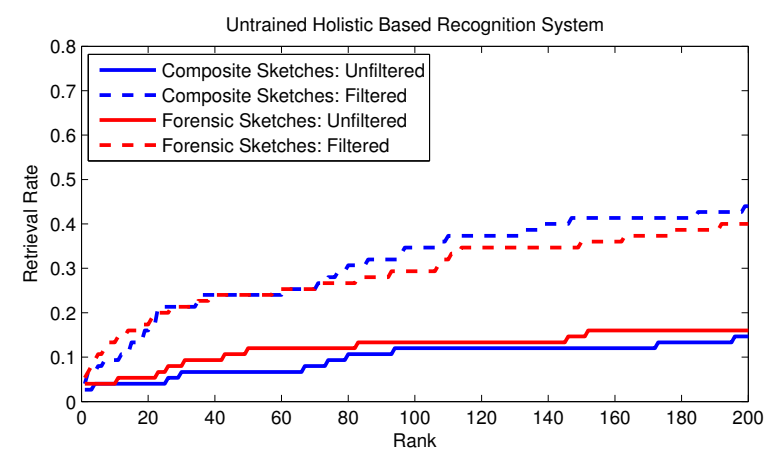

(a)

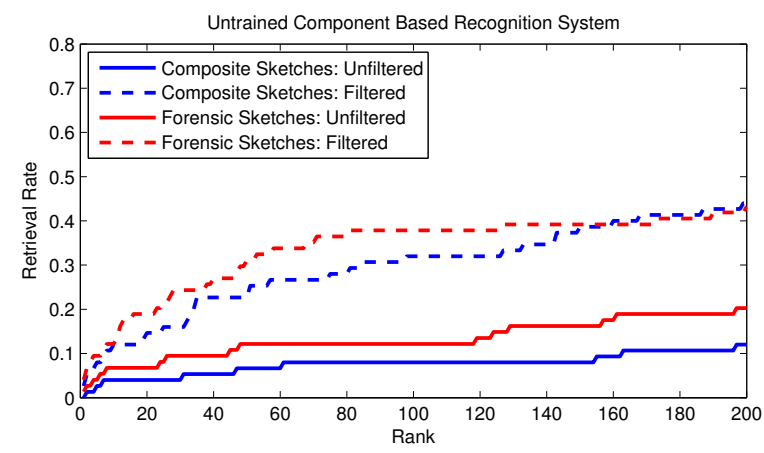

(c)

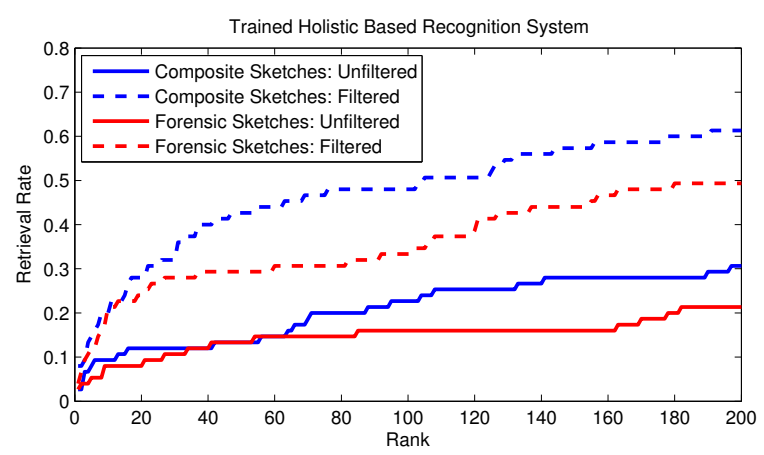

(b)

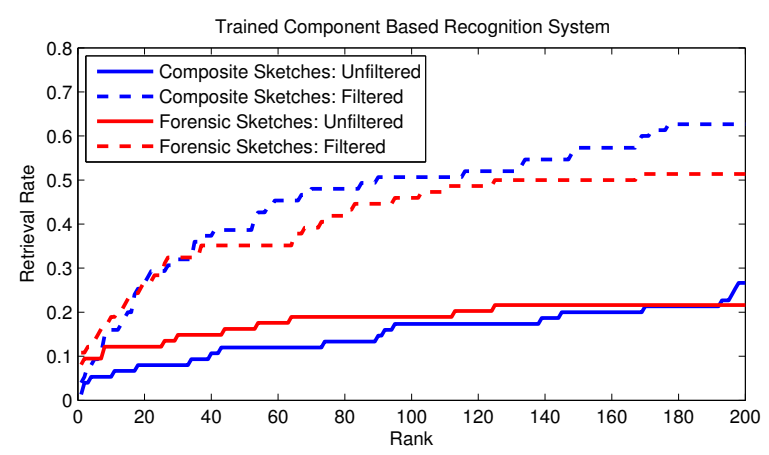

(d)

Figure 4: CMC plots for sketch to photo matching. Accuracies for the holistic method without training and with training can be found in (a) and (b). Accuracies for the component based method without and with training are shown in (c) and (d).

\section{Automatic facial sketch to photo matching}

Two facial sketch to photo matching algorithms were used in our study: a holistic method and a component based method. The holistic method, developed by Klare and Jain [10], uses similarities between local features computed on uniform patches across the entire face image. The individual patch scores are combined to calculate an overall sketch to mugshot match score. By contrast, the component based method by Han et al. [11] uses the similarity between individual facial components to compute an overall sketch to mugshot match score. Only a limited amount of training data in the form of successful matches of facial sketches to mugshots is available, namely (composite sketch, mugshot) and (forensic sketch, mugshot) mates. Therefore, we trained both methods using 606 (viewed sketch, photo) pairs used in [4]. Descriptions of these two matchers are given below.

\subsection{Holistic based face recognition}

The holistic method has been shown to be an effective technique for matching a facial sketch probe against a gallery of mugshots [10]. Following normalization, three filters are applied to both the probe sketch and the gallery image to compensate for the differences in modality. After tessellating a facial sketch/mugshot into 154 uniform patches, SIFT [27] and multi-scale local binary pattern (MLBP) [28] features are extracted from each patch. Training in the form of random sampling linear discriminant analysis (RS-LDA) [29] is used to improve the recognition accuracy. To measure the similarity between feature vectors, the holistic method uses the cosine similarity measure.

\subsection{Component based face recognition}

The component based method was proposed in [11] to match composite sketches to photos using two different facial composite systems (FACES and IdentiKit). In the component based method, facial components are automatically localized by detecting landmarks with an active shape model. A local descriptor-based representation, MLBP [28], is then utilized to capture the texture and structure at various scales in each facial component. The component based method uses linear discriminant analysis (LDA) to improve recognition accuracy. Block based feature extraction is employed to spatially encode the structural information of each facial component. The most accurate components to be used during score fusion are determined empirically for each sketch modality. This representation is consistent with the process of composite sketch synthesis in facial composite systems. However, the performance of the component based method on forensic sketches and non- 


\begin{tabular}{|c|c|c|c|c|c|c|}
\hline \multicolumn{7}{|c|}{ Males } \\
\hline \multirow{2}{*}{ Ethnicity } & $<20$ & $20-30$ & $30-40$ & $40-50$ & $50-60$ & $>60$ \\
& $<$ & 851 & 734 & 282 & 65 & 23 \\
\hline Black & 195 & 40 & 21 & 10 & 2 & 0 \\
Hispanic & 3 & 1983 & 2119 & 963 & 306 & 127 \\
White & 355 & 14 & 13 & 6 & 1 & 0 \\
Other & 4 & 14 & Females \\
\hline \hline \multicolumn{7}{|c|}{ Age Range } \\
\hline Ethnicity & $<20$ & $20-30$ & $30-40$ & $40-50$ & $50-60$ & $>60$ \\
\hline Black & 28 & 266 & 229 & 62 & 8 & 3 \\
Hispanic & 2 & 0 & 3 & 1 & 0 & 0 \\
White & 50 & 486 & 525 & 209 & 61 & 15 \\
Other & 0 & 4 & 4 & 2 & 0 & 0 \\
\hline
\end{tabular}

Table 2: Demographic information of the 10,075 subjects in the gallery set.

viewed composite sketches was not evaluated in [11].

\section{Experimental Results}

\subsection{Dataset and performance evaluation}

In total, our facial sketch dataset consists of 75 forensic sketches and 75 composite sketches with corresponding mugshots and demographic information. The true mugshot age was calculated to be the average of the age range provided by the volunteer while describing the suspect. To make our analysis more realistic, we extended the gallery to include 10,000 mugshots provided by the Pinellas County Sheriff's Office (PCSO), resulting in a gallery size of 10,075. Demographic information of the subjects in this dataset can be found in Table 2. Note that the other race/ethnicity contains all subjects not belonging to the black, hispanic, or white races/ethnicities. Filtering is not performed when encountering a probe with the other race/ethnicity. In forensic and biometrics scenarios involving facial sketch to mugshot matching, the standard procedure involves law enforcement officers looking through top- $N$ matches [2] (rather than only considering rank-one retrieval rates). In our experiments, $N=200$. We also used the performance of a commercial-off-the-shelf face matcher, FaceVACS v8.2 [19] as a baseline. As shown in Fig. 5, FaceVACS achieves rank-200 retrieval rates of $4.1 \%$ and $6.7 \%$ for forensic and composite sketches, respectively. The holistic and component representations (Fig. 4) each have significantly better performance compared to FaceVACS (Fig. 5) for both forensic and composite sketches under both the filtered and unfiltered scenarios.

\subsection{Holistic method: forensic vs. composite}

The holistic recognition method performs comparably for both forensic sketches and composite sketches without training (Fig. 4(a)). Interestingly, training (Fig. 4(b)) improves the rank-200 retrieval rates for composite sketches

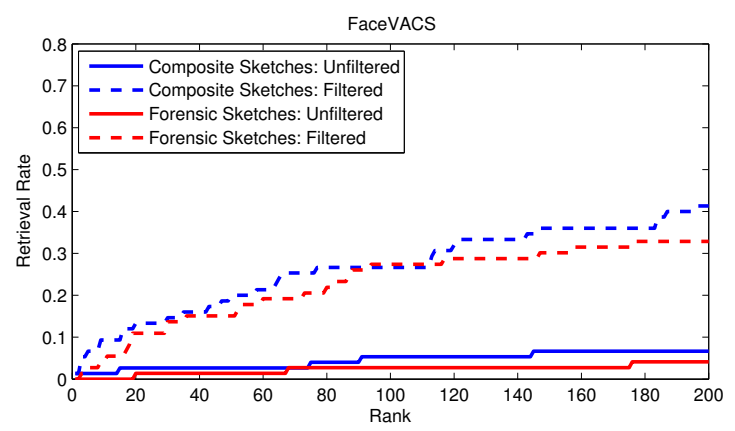

Figure 5: CMC plots for matching facial sketches to mugshots using the commercial matcher FaceVACS.

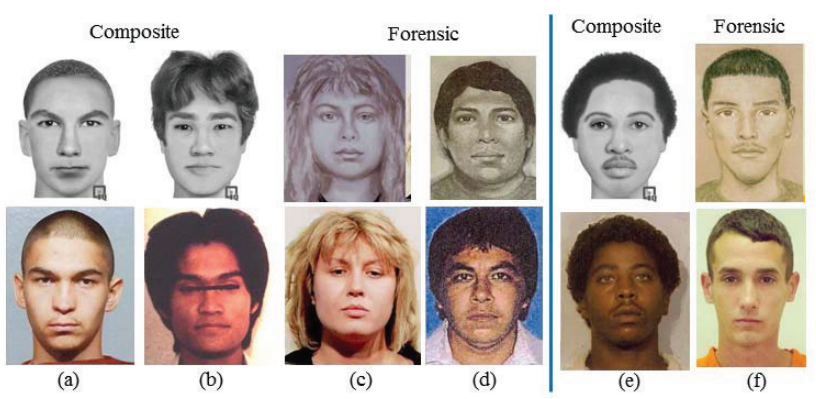

Figure 6: Examples of sketch to mugshot matching using the component method with demographic filtering. (ad): Successful matches for composite sketch and forensic sketch, where the true mugshot is matched at rank-1. (ef): Poor matches for composite sketch and forensic sketch, where the true mugshot is matched beyond rank-500.

(14.67\% without training to $30.67 \%$ with training) more significantly than retrieval rates for the forensic sketches ( $16.0 \%$ without training to $21.33 \%$ with training). This suggests that the viewed sketches used for training more closely resemble the modality of the composite sketches.

\subsection{Component method: forensic vs. composite}

The component based method improves upon the performance of the holistic method in matching forensic sketches when neither system has been trained (Fig. 4(c)). The retrieval rate for composite sketches is worse due to the fact that most components are inaccurate (similar to Fig. 7(a)). After training (Fig. 4(d)), the rank-200 retrieval rate of the component based method is nearly identical to that of the holistic method for composite sketches and is worse than the holistic method for forensic sketches. This result is perhaps expected, since the training method employed by the holistic method (RS-LDA) is more advanced than the training method used by the component based method (LDA). Similar to the holistic method, training the component based method improves the retrieval rate of composite sketches ( $12.00 \%$ without training to $26.67 \%$ with training) more than that of forensic sketches $(20.27 \%$ without training to $21.62 \%$ with training). 


\begin{tabular}{ccccc}
\multirow{2}{*}{ Face Matcher } & \multirow{3}{c}{ Sketch Type } & $10.0 \%$ & $1.0 \%$ & $0.1 \%$ \\
\hline \multirow{2}{*}{ Holistic } & Forensic & 85.33 & 25.33 & 8.00 \\
& Composite & 94.67 & 38.67 & 4.00 \\
\hline \multirow{2}{*}{ Component } & Forensic & 82.43 & 31.08 & 16.22 \\
& Composite & 86.67 & 33.33 & 9.33 \\
\hline \multirow{2}{*}{ FaceVACS } & Forensic & 72.60 & 8.22 & 1.37 \\
& Composite & 82.67 & 18.67 & 2.67 \\
\hline
\end{tabular}

Table 3: True accept rates (TAR) at false accept rates (FAR) of $10 \%, 1 \%$ and $0.1 \%$ for the three face matchers after filtering. The holistic and component based methods used here were trained on the CUHK dataset (http://mmlab.ie.cuhk.edu.hk/facesketch.html).

\subsection{Filtering with demographic information}

Filtering the mugshot gallery using gender, age range, and race/ethnicity significantly improves retrieval rates of all three matchers. While this was also shown in an earlier study [9], that study only used gender and race/ethnicity for filtering. Our experimental results indicate that age range is the strongest of the three demographic filters. Since a suspect's demographic information is usually easy for a witness to provide, we consider it to be a powerful technique for improving the recognition accuracy. Considering the trained holistic method, the rank-200 retrieval rate increases from $21.33 \%$ to $49.33 \%$ and from $30.67 \%$ to $61.33 \%$ for forensic and composite sketches, respectively. The increase in retrieval rate using the trained component method is similarly large: $21.62 \%$ to $51.35 \%$ and $26.67 \%$ to $62.67 \%$ for forensic and composite sketches, respectively. We have included true accept rates (TAR) at various false accept rates (FAR) (Table 3) so that other published methods can be compared against ours.

It is worth noting that filtering improves the retrieval rate for composite sketches more than that of forensic sketches. This is due to the nature of composite sketch synthesis, in which individual facial components are selected. While creating a composite sketch, it is sometimes the case that a selected component belongs to a demographic group different than the corresponding mugshot (i.e., when the lips selected for the composite sketch are androgynous but the mugshot is male). During matching, these components increase the similarity score of composite sketches and mugshots outside the correct demographic.

There is also a noticeable improvement in retrieval rate after filtering for the component method compared with the holistic method. This is due to the fact that before filtering, most components provide very poor recognition accuracy individually, so there is little benefit derived from their fusion (Fig. 7(a)). After filtering, the recognition accuracy of each component is significantly improved leading the component fusion accuracy to be much higher (Fig. 7(b)). Examples of good matches and poor matches using the com-

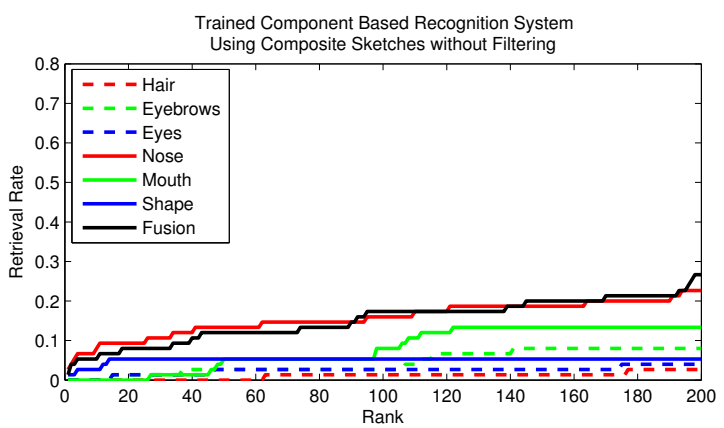

(a)

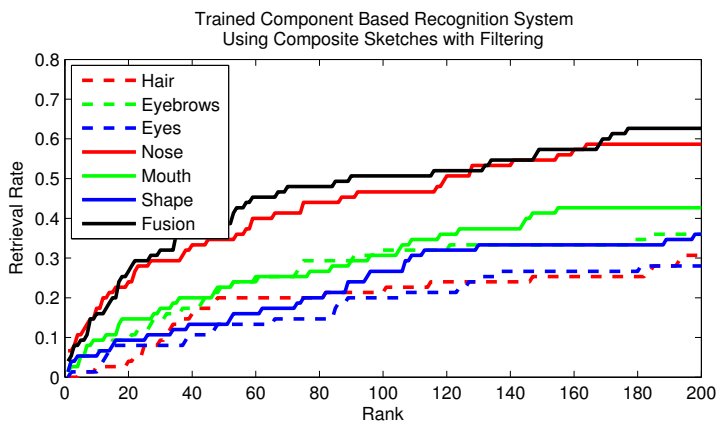

(b)

Figure 7: Component based recognition accuracies before (a) and after (b) filtering with demographic information. The increase in individual component accuracies improves the fused retrieval rate drastically.

ponent method with demographic filtering can be found in Fig. 6.

Interestingly, the fusion of components only achieves a slight accuracy improvement over the performance of the nose component alone. The reason for this is that most volunteers are able to provide a detailed description of the nose, such as the width of the base and shape of the nostrils. Additionally, there are many options (around 1,200) for the nose component in FACES. As a result, the witness is able to provide a more accurate description of the nose compared to other facial components.

\section{Summary}

Facial sketches drawn by forensic artists (forensic sketches) or created using software (composite sketches) are used by law enforcement agencies to assist in identification and apprehension of suspects involved in criminal activities. Using a new dataset of 75 forensic sketches and 75 composite sketches (available at http: / / biomet rics.cse.msu.edu/pubs/databases.html), we have performed the first comparison of the effectiveness of both modalities when used with two state-of-the-art sketchto-photo matchers. A commercial face matcher was also used as a baseline. Demographic information was further 
explored to filter the gallery (10,075 mugshots) to improve the matching performance. Composite sketches were found to be more effective in identifying the suspect's mugshots. In our future work, we plan to enlarge the composite sketch dataset to 200 by creating composite sketches for additional mugshots that have mated forensic sketches.

\section{Acknowledgement}

The authors would like to thank Scott McCallum and his team at the Pinellas County Sheriff's Office for their support of this research. We would also like to thank Lois Gibson and the sketch artists from the Michigan State Police for their contribution of data to this work. This research was supported by National Institute of Justice (NIJ) grant 2011IJ-CX-K057.

\section{References}

[1] FACES 4.0, IQ Biometrics, http://www.iqbiometrix.com.

[2] A. Jain, B. Klare, and U. Park, "Face matching and retrieval in forensics applications," IEEE Multimedia, vol. 19, no. 1, pp. 20-28, 2012.

[3] D. Mcquiston, L. Topp, and R. Malpass, "Use of facial composite systems in US law enforcement agencies," Psychology, Crime and Law, vol. 12, no. 5, pp. 505-517, 2006.

[4] X. Tang and X. Wang, "Face sketch recognition," IEEE Trans. on Circuits and Systems for Video Technology, vol. 14, pp. 50 - 57, January 2004.

[5] Q. Liu, X. Tang, H. Jin, H. Lu, and S. Ma, "A nonlinear approach for face sketch synthesis and recognition," Proc. IEEE Conf. Computer Vision and Pattern Recognition, pp. 1005-1010, June 2005.

[6] D. Lin and X. Tang, "Inter-modality face recognition," Proc. ECCV, pp. 13-26, 2006.

[7] P. Yuen and C. Man, "Human face image searching system using sketches," IEEE Trans. SMC, Part A: Systems and Humans, vol. 37, pp. 493-504, July 2007.

[8] X. Wang and X. Tang, "Face photo-sketch synthesis and recognition," IEEE Trans. on Pattern Analysis and Machine Intelligence, vol. 31, pp. 1955-1967, November 2009.

[9] B. Klare, Z. Li, and A. Jain, "Matching forensic sketches to mug shot photos," IEEE Trans. Pattern Analysis and Machine Intelligence, vol. 33, pp. 639-646, March 2011.

[10] B. Klare and A. Jain, "Heterogeneous face recognition using kernel prototype similarities," IEEE Trans. on Pattern Analysis and Machine Intelligence, 2013 (to appear).

[11] H. Han, B. Klare, K. Bonnen, and A. Jain, "Matching composite sketches to face photos: A component based approach," IEEE Trans. on Information Forensics and Security, vol. 8, pp. 191-204, January 2013.

[12] C. Frowd, D. Carson, H. Ness, D. McQuiston, J. Richardson, H. Baldwin, and P. Hancock, "Contemporary composite techniques: The impact of a forensically-relevant target delay," Legal and Criminological Psychology, vol. 10, pp. 6381, February 2005.

[13] C. Koehn and R. Fisher, "Constructing facial composites with the mac-a-mug pro system," Psychology, Crime and Law, vol. 3, no. 3, pp. 215-224, 1997.

[14] V. Bruce, H. Ness, P. Hancock, C. Newman, and J. Rarity, "Four heads are better than one: Combining face composites yields improvements in face likeness," Journal of Applied Psychology, vol. 87, pp. 894-902, October 2002.

[15] X. Gao, J. Zhong, and C. Tian, "Face sketch synthesis algorithm based on e-hmm and selective ensemble," IEEE Trans. on Circuits and Systems for Video Technology, vol. 18, pp. 487-496, April 2008.

[16] Y. Zhang, C. McCullough, J. Sullins, and C. Ross, "Handdrawn face sketch recognition by humans and a pca-based algorithm for forensic applications," IEEE Trans. SMC, Part A: Systems and Humans, vol. 40, pp. 475-485, May 2010.

[17] R. Uhl and N. Lobo, "A framework for recognizing a facial image from a police sketch," in Proc. IEEE Conf. Computer Vision and Pattern Recognition, pp. 586-593, June 1996.

[18] H. Bhatt, S. Bharadwaj, R. Singh, and M. Vatsa, "Memetically optimized mcwld for matching sketches with digital face images," IEEE Trans. on Information Forensics and Security, vol. 7, pp. 1522-1535, October 2012.

[19] FaceVACS Software Developer Kit v. 8.2, Cognitec Systems $\mathrm{GmbH}, \mathrm{http}: / / \mathrm{www} . \operatorname{cog} n i t e c-s y s t e m s . d e$.

[20] S. Li and A. Jain (eds.), Handbook of Face Recognition. Springer, second ed., 2011.

[21] L. Gibson, Forensic Art Essentials. Elsevier, 2008.

[22] E-FIT, VisionMetric Ltd., http://www.vision-metric.com.

[23] C. Frowd, P. Hancock, and D. Carson, "Evofit: A holistic, evolutionary facial imaging technique for creating composites," ACM Trans. on Applied Psychology, vol. 1, pp. 19-39, July 2004.

[24] Identi-Kit, Identi-Kit Solutions, http://www.identikit.net/.

[25] G. Wells and L. Hasel, "Facial composite production by eyewitnesses," Current Directions in Psychological Science, vol. 16, pp. 6-10, February 2007.

[26] R. Geiselman, R. Fisher, D. MacKinnon, and H. Holland, "Eyewitness memory enhancement with the cognitive interview," American Journal of Psychology, vol. 99, pp. 385401, 1986.

[27] D. Lowe, "Distinctive image features from scale-invariant keypoints," Int. Journal of Computer Vision, vol. 60, no. 2, pp. 91-110, 2004.

[28] T. Ojala, M. Pietikäinen, and T. Mäenpää, "Multiresolution grayscale and rotation invariant texture classification with local binary patterns," IEEE Trans. on Pattern Analysis and Machine Intelligence, vol. 24, no. 7, pp. 971-987, 2002.

[29] X. Wang and X. Tang, "Random sampling for subspace face recognition," Int. Journal of Computer Vision, vol. 70, no. 1, pp. 91-104, 2006. 